\title{
Integrin Receptor Antagonist GLPG0187
}

National Cancer Institute

\section{Source}

National Cancer Institute. Integrin Receptor Antagonist GLPG0187. NCI Thesaurus. Code C96223.

A small molecule integ rin receptor antagonist (IRA) with potential antineoplastic activity. Upon administration, GLPG0187 binds to and blocks the activity of 5 RGD-integrin receptor subtypes, including alphavbeta1, alphavbeta3, alphavbeta5, alphavbeta6 and alpha5beta1. This may result in the inhibition of endothelial cell-cell interactions and endothelial cell-matrix interactions, and the prevention of angiogenesis and metastasis in tumor cells expressing these integrin receptors. Integrin receptors are transmembrane glycoproteins expressed on the surface of tumor vessel endothelial cells and some types of cancer cells, and play a crucial role in endothelial cell adhesion and migration. 